Sādhanā Vol. 39, Part 6, December 2014, pp. 1425-1446. (C) Indian Academy of Sciences

\title{
Simulation of experimental breakthrough curves using multiprocess non-equilibrium model for reactive solute transport in stratified porous media
}

\author{
DEEPAK SWAMI, P K SHARMA* and C S P OJHA \\ Department of Civil Engineering, Indian Institute of Technology Roorkee, \\ Roorkee 247 667, India \\ e-mail: drpksharma07@gmail.com
}

MS received 25 January 2014; revised 9 July 2014; accepted 20 July 2014

\begin{abstract}
In this paper, we have studied the behaviour of reactive solute transport through stratified porous medium under the influence of multi-process nonequilibrium transport model. Various experiments were carried out in the laboratory and the experimental breakthrough curves were observed at spatially placed sampling points for stratified porous medium. Batch sorption studies were also performed to estimate the sorption parameters of the material used in stratified aquifer system. The effects of distance dependent dispersion and tailing are visible in the experimental breakthrough curves. The presence of physical and chemical non-equilibrium are observed from the pattern of breakthrough curves. Multi-process non-equilibrium model represents the combined effect of physical and chemical non-ideality in the stratified aquifer system. The results show that the incorporation of distance dependent dispersivity in multi-process non-equilibrium model provides best fit of observed data through stratified porous media. Also, the exponential distance dependent dispersivity is more suitable for large distances and at small distances, linear or constant dispersivity function can be considered for simulating reactive solute in stratified porous medium.
\end{abstract}

Keywords. Solute transport experiment; stratified porous medium; breakthrough curves; dispersion; sorption.

\section{Introduction}

The transport of contaminant through porous media is a very complex process and it is dominated by several physical and chemical factors related to porous medium. Any variations in the physical and chemical properties of the porous medium induce an imbalance in the transport of contaminant. For reducing the complexity of transport, some assumptions are employed. The hypothesis that has been used widely in solute transport to reduce its complexity is that the aquifer properties are invariant and local equilibrium assumption is valid during the transport (Freeze \& Cherry

${ }^{*}$ For correspondence 
1979). In the field conditions at a given velocity, these assumptions sustain an ideal transport behaviour. However, there are many macro and micro level processes which influence the applicability of ideal transport. Practically the equilibrium assumption does not exist in the real field conditions and the nature of the solute transport is always in non-equilibrium regime. The nonequilibrium transport of contaminant is reported in the literature (Van Genuchten \& Wierenga 1976; Brusseau \& Rao 1989; Roberts et al 1986; Rao et al 1980). Solute transport under nonequilibrium condition is differentiated on the basis of the factors inducing the non-equilibrium, either physical or chemical. Physical non-equilibrium influences the solute transport in the presence of couples of spatially varying hydraulic conductivity. Chemical non-equilibrium is present because of site-specific rate of chemical interactions between aquifer material and solute particles. In the initial investigations, physical non-equilibrium was reported by several researchers in the formation of mobile-immobile coupling. Generally, the behaviour of the transport of contaminant through porous medium is represented by breakthrough curves. Breakthrough curves are the relation between normalized concentration $\left(\mathrm{C} / \mathrm{C}_{0}\right)$ or concentration with time, obtained at any distance in flow direction. The shape of breakthrough curves can be used to understand the transport behaviour. Skewed shaped breakthrough encouraged many researchers to develop the theories related to physical and chemical non-equilibrium.

Laboratory and field scale experiments support the theory of physical non-equilibrium (Van Genuchten \& Wierenga 1976; Gillham et al 1984; Sudicky et al 1985; Starr et al 1985; Van Duijn \& van derZee 1986; Liu \& Bing 2008). Sudicky et al (1985) and Starr et al (1985) observed the behaviour of breakthrough curves from the elution of non-reactive and reactive solute through stratified porous medium. The nature of breakthrough curves showed a significant departure from classical dispersion-diffusion theory and non-fickian behaviour of diffusion was observed. It was found that there are some physio-chemical interactions influencing the transport of reactive contaminant which is not explained by classical theory (Starr et al 1985). The distribution of breakthrough curves resulting from the elution of solute through porous medium under the influence of non-equilibrium condition is asymmetrical. There are different conceptual models proposed by several field experiments to simulate such irregular shape of breakthrough curves. Van Genuchten \& Wierenga (1976) developed mobile-immobile model (MIM) which was based on practical and physical approach to describe the contaminant transport behaviour in heterogeneous soil. The MIM model considers stagnant domain in the porous media as well as exchange between mobile and stagnant domains. Van Genuchten (1981) developed analytical solutions for one-dimensional advective-dispersive transport equation considering linear equilibrium adsorption and first order decay. Three-dimensional solutions for solute transport in an infinite porous medium considering whole porous medium in the several distributed discrete mobile-immobile zones to observe the tailing effect of breakthrough curve was presented by Goltz \& Roberts (1986). The results obtained by several laboratory experiments could not fit properly using numerical model based on bicontinuum model concept. There were still discrepancies found between the simulation and the observed data. It is assumed that the conditions of local equilibrium existed during solute transport under natural conditions; however solute transport is influenced by non-equilibrium (Brusseau \& Rao 1989). Brusseau et al (1989, 1992) presented an advective-dispersive transport model that explicitly accounts for multiple sources of non-equilibrium and transformation reactions during steady state flow in the porous media. This transport model has been used for simulation of experimental data for reactive plume at field scale and laboratory scale. The capability of MPNE model to predict the transport of solute transport through porous medium has been evaluated by Brusseau $(1991,1992)$ and Burr et al (1994). Analytical solution of MPNE model was developed by Xu \& Brusseau (1996) adding spatial variability in transformation reactions adds more flexibility in the model. 
Elzein \& Booker (1999) presented boundary element method to solve multiple sources of nonequilibrium processes in saturated heterogeneous media and validated the results with analytical solution. The effect of non-ideal transport is well-discussed for reactive contaminant. The effect of Physical non-equilibrium can be understood by dividing the porous medium in mobile and immobile domains, but to understand the effect of chemical non-equilibrium, in depth analysis of sorption site is required. Naik et al (2008) examined the effective diffusion coefficient of sodium in the presence of sulphate using column test. As the retardation of ions increases, the difference between theoretical and experimental curves of diffusivity increases. Khan et al (2010) studied the adsorption of $\mathrm{Cr}(\mathrm{VI})$ from aqueous solution by soil in both batch and continuous mode of operations.

Even after considering such detailed processes in multiprocess non-equilibrium (MPNE), it is obvious that the inclusion of the scale dependent function of dispersivity is needed. Statistical variations in the physical properties of porous medium and the scale of measurement can results in scale dependent dispersivity function (Pickens \& Grisak 1981). Scale dependent dispersivity has been analysed extensively in the literature (Molz et al 1983; Roberts et al 1986; Barry \& Sposito 1989; Yates 1990, 1992). Scale dependent dispersivity combining with dual porosity system is found to provide more precise results to the observed data obtained from non-ideal transport (Dykhuizen 1991; Gerke \& Van Genuchten 1996; Huang et al 2006; Gao et al 2010).

Transport of reactive contaminant through stratified porous media can be represented more accurately by considering multiprocess non-equilibrium model. MPNE model considers all possible reactions in the forms of representing parameters such as equilibrium sorption, mass transfer and reverse sorption coefficients. Physical and chemical non-equilibrium are the lumped terms which include all the micro reactions influencing the system. The application of the MPNE model provides advantage to use discrete kinetic terms for each of the reaction that yields the advantage of process level investigation. The basic advantage of MPNE model is to provide the details of the system processes independently rather than a lumped kinetic term.

Physical non-equilibrium (PNE) can be introduced in the system by the combination of advective and non-advective regions connected hydraulically. Advective and non-advective regions are also known as mobile-immobile regions prominently. Due to the difference in the hydraulic conductivities, a fraction of mass transferred in the non-advective region driven by diffusive gradient. Because of minimum flow through immobile region, it is behaving as a distributed sink/source components. Early initial appearance of contaminant and long tailing effect in breakthrough curve is occurred under the physical non-equilibrium conditions. Chemical non-equilibrium (CNE) is a microscopic reaction which exists in the system due to chemical interaction between the solute particles and grains of porous medium. Intraparticle diffusion and rate limited interaction between solute and specific sorption sites and intrasorbent diffusion are the basic reaction involved in producing the chemical non-equilibrium. Low polarity, hydrophobic, organic chemicals are generally site independent and do not participate in chemical non-equilibrium (Chiou et al 1979; Karickhoff 1981; Brusseau \& Rao 1989). Transport of non-hydrophobic and all inorganic solutes are generally influenced by chemical non-equilibrium.

Chemical non-equilibrium (CNE) occurs in the transport of solute inherently, whereas in the laboratory physical non-equilibrium (PNE) is introduced by combining two different materials with different hydraulic conductivity.

The sorption characteristics of the sites are also very important in the system influenced by chemical non-equilibrium. The site-specific sorption becomes dominating at lesser velocity and when the system is under the influence of mobile-immobile partitioning. Spreading at microscopic level is basically influenced by heterogeneity and concentration gradients. Because of higher adsorption, solute particles get accumulated around grains and intraparticle diffusion start 
participating in non-equilibrium (Brusseau et al 1989). The impact of site-specific sorption is quantitatively incorporated in retardation factor, and can be calculated in the laboratory by batch sorption test.

In this study, an attempt has been made to investigate the behaviour of reactive (sorbing) solute transport through stratified porous media under the influence of multiprocess nonequilibrium. Multiprocess non-equilibrium (MPNE) parameters are estimated while fitting the observed data by employing linear sorption isotherm. To improve the results, the distance dependent dispersivity function is considered. Constant, linear and exponential distance dependent dispersivity functions are then compared in the breakthrough curves.

\section{Conceptual framework and formulation}

The experiments performed by Sudicky et al (1985) and Starr et al (1985) on stratified porous medium showed the impact of interlayer diffusive mass transfer. The simulation results showed a fair presentation of observed data, though the retardation factor was needed to optimize. The value of retardation factor calculated by equilibrium sorption coefficient was much greater than the optimized values. So it is suggested that some factors are present in the system which will affect the transport of solute through the stratified porous medium. Later, Brusseau (1991) tried to simulate the data set using MPNE model and found more satisfying results. The micro aquifer system used in the laboratory was short in travel distance (Sudicky et al 1985; Starr et al 1985). But it is seen that if the length of the model is appreciable, then apart from the inclusion of MPNE model, distance dependent dispersivity can enhance the simulation. Since the system is under the influence of physical and chemical non-equilibrium, multiprocess non-equilibrium with distance dependent dispersivity can provide a better fit to the experimental data.

Tang et al (1981) presented the coupling between the fracture and the porous matrix for the similar condition by continuity of fluxes and concentration along the interface. The identical fate of transport in stratified porous media can be considered by assigning a value of porosity of fracture medium equal to that of the advective layer. It is assumed that diffusive flux from the sand layer to the porous matrix takes place perpendicular to the stratification and complete mixing across the stratification takes place all the time.

For porous formation, Brusseau (1992) developed a general transport equation that account for both physical and chemical non-equilibrium. An advective-dispersive-reactive transport equation for transport of reactive solute through the advective region of porous media can be written as

$$
\begin{aligned}
\left(\theta_{\mathrm{a}}+f \rho F_{\mathrm{a}} K_{\mathrm{a}}\right) \frac{\partial C_{a}}{\partial t}+f \rho \frac{\partial S_{\mathrm{a} 2}}{\partial t}= & \theta_{\mathrm{a}} \frac{\partial}{\partial x}\left(D(x) \frac{\partial C_{\mathrm{a}}}{\partial x}\right)-\theta_{\mathrm{a}} v_{\mathrm{a}} \frac{\partial C_{\mathrm{a}}}{\partial x}-\omega\left(C_{\mathrm{a}}-C_{\mathrm{n}}\right) \\
& -\left[\mu_{\mathrm{a}} \theta_{\mathrm{a}}+\mu_{\mathrm{a} 1} f \rho F_{\mathrm{a}} K_{\mathrm{a}}\right] C_{\mathrm{a}}-\mu_{\mathrm{a} 2} f \rho S_{\mathrm{a} 2} .
\end{aligned}
$$

The mass balance for non-advective region can be written as

$$
\begin{aligned}
{\left[\theta_{\mathrm{n}}(1-f) \rho F_{\mathrm{n}} K_{\mathrm{n}}\right] \frac{\partial C_{\mathrm{n}}}{\partial t}+(1-f) \rho \frac{\partial S_{\mathrm{n} 2}}{\partial t}=} & \omega\left(C_{\mathrm{a}}-C_{\mathrm{n}}\right)-\left[\mu_{\mathrm{n}} \theta_{\mathrm{n}}+\mu_{\mathrm{n} 1}(1-f)\right. \\
& \left.\times \rho F_{\mathrm{n}} K_{\mathrm{n}}\right] C_{\mathrm{n}}-\mu_{n 2}(1-f) \rho S_{\mathrm{n} 2},
\end{aligned}
$$

where $\theta_{\mathrm{a}}$ is the fractional volumetric water content of advective region, $\left(\mathrm{L}^{3} / \mathrm{L}^{3}\right) ; \theta_{\mathrm{n}}$ is the fractional volumetric water content of the non-advective region, $\left(\mathrm{L}^{3} / \mathrm{L}^{3}\right) ; \rho$ is the bulk density of the porous medium, $\left(\mathrm{M} / \mathrm{L}^{3}\right) ; F_{\mathrm{a}}, F_{\mathrm{n}}$ are the mass fractions of sorbent for which sorption is 
essentially instantaneous; $K_{\mathrm{a}}, K_{\mathrm{n}}$ are the equilibrium sorption coefficient for advective and nonadvective region, $\left(\mathrm{L}^{3} / \mathrm{M}\right) ; f$ is the mass fraction of sorbent comprising the mobile region; $C_{\mathrm{a}}$ Concentration of solute in solution phase of advective region, $\left(\mathrm{M} / \mathrm{L}^{3}\right) ; C_{\mathrm{n}}$ Concentration of solute in solution phase of non-advective region, $\left(\mathrm{M} / \mathrm{L}^{3}\right) ; S_{\mathrm{a} 2}, S_{\mathrm{n} 2}$ are the rate limited sorbed phase concentration in advective and non-advective regions, $(\mathrm{M} / \mathrm{M}) ; D(x)$ is the hydrodynamic dispersion coefficient as function of distance, $\left(\mathrm{L}^{2} / \mathrm{T}\right) ; q$ is the specific discharge (equal to $v_{a} * \theta_{\mathrm{a}}$ ), $(\mathrm{L} / \mathrm{T}) ; v_{\mathrm{a}}$ is the pore water velocity in advective region, $(\mathrm{L} / \mathrm{T}) ; \omega$ is the first order coefficient of mass transfer between advective and non-advective region, $\left(\mathrm{T}^{-1}\right) ; \mu_{\mathrm{a}}$ and $\mu_{\mathrm{n}}$ are the first-order transformation coefficients for the solution phase in advective and non-advective regions, $\left(\mathrm{T}^{-1}\right)$; $\mu_{\mathrm{a} 1}$ and $\mu_{\mathrm{n} 1}$ are the transformation coefficients $\left(\mathrm{T}^{-1}\right)$ for the instantaneous sorbed-phase of advective and non-advective regions, respectively, and $\mu_{\mathrm{a} 2}$ and $\mu_{\mathrm{n} 2}$ are the transformation coefficients for rate-limited sorbed-phase domains of advective and non-advective regions, respectively.

Dynamics of sorption and transformation for the rate limited domains are described by:

$$
\begin{gathered}
\frac{\partial S_{\mathrm{a} 2}}{\partial t}=k_{\mathrm{a} 2}\left[\left(1-F_{\mathrm{a}}\right) K_{\mathrm{a}} C_{\mathrm{a}}-S_{\mathrm{a} 2}\right]-\mu_{\mathrm{a} 2} S_{\mathrm{a} 2}, \\
\frac{\partial S_{\mathrm{n} 2}}{\partial t}=k_{\mathrm{n} 2}\left[\left(1-F_{\mathrm{n}}\right) K_{\mathrm{n}} C_{\mathrm{n}}-S_{\mathrm{n} 2}\right]-\mu_{\mathrm{n} 2} S_{\mathrm{n} 2},
\end{gathered}
$$

where $k_{\mathrm{a} 2}$ and $k_{\mathrm{n} 2}$ are the first order reverse sorption coefficients $\left(\mathrm{T}^{-1}\right)$ for the advective and non-advective regions, respectively.

Following initial and pulse type boundary conditions are used:

$$
\begin{gathered}
C_{\mathrm{a}}(x, 0)=C_{\mathrm{n}}(x, 0)=0, \\
S_{\mathrm{a} 2}(x, 0)=\mathrm{S}_{\mathrm{n} 2}(x, 0)=0, \\
C_{\mathrm{a}}(0, t)=C_{0} \quad 0<t<T_{0}, \\
C_{\mathrm{a}}(0, t)=0 \quad t>T_{0},
\end{gathered}
$$

where $T_{0}$ is the pulse duration.

Implementing above boundary conditions, hydrodynamic dispersion coefficient can be represented as (Yates 1990):

$$
D(x)=\alpha(x) v_{\mathrm{a}}+D_{0} .
$$

In above equation, the dispersivity is considered as a function of distance $\mathrm{x}$, which is the distance travelled by solute plume from the origin. The dispersivity $\alpha(x)$ can be considered as constant, linear, and exponential function of distance $\mathrm{x}$. The distance dependent functions of dispersivity are described and explained below.

\subsection{Linear distance dependent function}

Under the consideration of this function, dispersivity increases linearly with the increase of the distance of solute from front to origin. Under this assumption, at infinite distance the dispersivity will attain an infinite value. The relation is given by Yates (1990):

$$
\alpha(x)=k x
$$

where $\mathrm{k}$ is the coefficient of distance dependent dispersivity and $\mathrm{x}$ is the distance $(\mathrm{L})$. 


\subsection{The second type of function is Exponential distance dependent}

This type is considered to be more realistic. In linear function, the dispersivity increases without bounds with distance and due to this higher values can be obtained at large distance in the flow direction. Exponential function initially increases with distance and attains an asymptotic value at large distance which is ethically accepted. The exponential distance dependent dispersivity is described as (Yates 1992):

$$
\alpha(x)=a_{1}\left(1-e^{-b_{1} x}\right),
$$

where $a_{1}$, is the asymptotic value (L) and $b_{1}$ is a positive constant $\left(\mathrm{L}^{-1}\right)$.

Constant dispersivity is also considered in this study. The value of constant dispersivity is estimated by convection dispersion equation (CDE) and considered as a space independent input.

Finite difference method utilizing Crank-Nicholson technique is used to solve the above sets of transport Eqs. (1-4). FORTRAN numerical code is developed and simulation is done for the observed experimental data through stratified porous media. The procedure of experiments and material properties is discussed in following section.

\section{Material properties}

The particle size distribution and hydraulic conductivity of fine sand and silt are examined prior to performing the experiments. Porous material is basically classified by the mean grains diameter. Porous material is used in the experiments having different grain size distribution and hydraulic conductivity. The distribution grain size of soil media is shown in figure 1 . The estimated value of physical properties of soil media is shown in table 1.

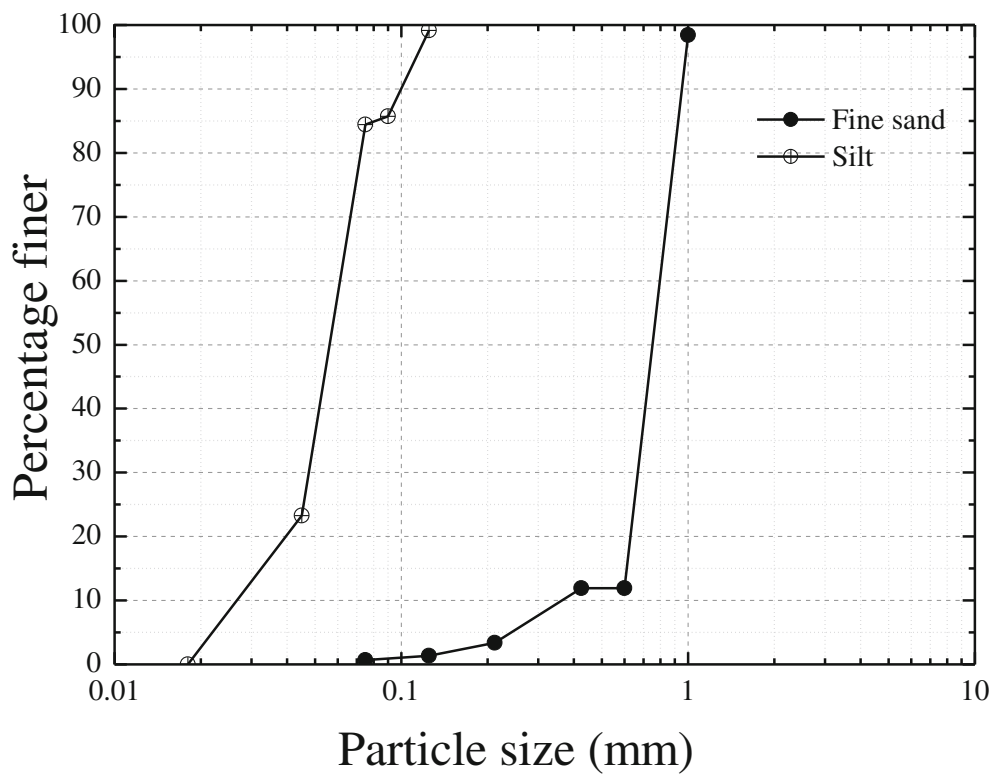

Figure 1. Particle size distribution curve of soil used in the experiment. 
Table 1. Physical properties of soil media.

\begin{tabular}{|c|c|c|c|c|c|c|c|c|}
\hline Material & $\begin{array}{c}\text { Bulk } \\
\text { density } \\
\left(\mathrm{g} / \mathrm{cm}^{3}\right)\end{array}$ & Porosity & $\begin{array}{l}\text { Hydraulic } \\
\text { conductivity } \\
\text { (m/day) }\end{array}$ & $\begin{array}{c}\mathrm{C}_{c} \\
(\mathrm{~mm})\end{array}$ & $\mathrm{C}_{u}$ & $\mathrm{D}_{10}$ & $\begin{array}{c}\text { Mean grain } \\
\text { Size }\left(\mathrm{D}_{50}\right) \\
(\mathrm{mm})\end{array}$ & Method \\
\hline Silt & 1.63 & 0.4 & Order of $10^{-6}$ & 1.213 & 2.81 & 0.026 & 0.055 & $\begin{array}{l}\text { Bulk density was measured } \\
\text { with sand replacement } \\
\text { method, hydraulic conductivity } \\
\text { was measured with falling head }\end{array}$ \\
\hline Fine sand & 1.82 & 0.32 & Order of $10^{-1}$ & 1.56 & 2.16 & 0.057 & 0.75 & $\begin{array}{l}\text { method, Mean grain sizes were } \\
\text { calculated by mechanical sieve } \\
\text { analysis }\end{array}$ \\
\hline
\end{tabular}

It is seen that the value of hydraulic conductivity of fine sand is higher as compared to silt. A higher value of hydraulic conductivity ensures easier flow path for displacing of fluid, while lesser hydraulic conductivity ensures immobility for the flow. These combinations provide a mobile-immobile model to investigate the fate of contaminant under controlled laboratory conditions. The soil media chosen are from different sites, and all are free from any type of contamination. Fine sand was obtained from the upper bank of river Ganga which was free from any chemicals and well-sorted particles. The silt was obtained from Irrigation Research Institute, Bahadarabad. Before performing the experiments, it was washed for 2 to $4 \mathrm{~h}$ with fresh water in a container to remove the initial concentration of chemical, to drain out water only by providing $0.45 \mu \mathrm{m}$ cellulose acetate syringe filter cover at bottom outlet.

\section{Batch experiments}

Sorption isotherm for fluoride was conducted in $500 \mathrm{ml}$ glass container at room temperature. Blank samples with no added $\mathrm{NaF}$ were also included in the experiments to verify whether or not natural background fluoride was desorbing from the soil. The sample container containing 5, 10 and $15 \mathrm{~g}$ of material in $300 \mathrm{ml}$ of fluoride solution were shaken for $24 \mathrm{~h}$ and then samples were filtered using a $0.45 \mu \mathrm{m}$ cellulose acetate syringe filter and analysed for fluoride concentration. The amount of fluoride adsorbed by soil was calculated from the difference between the final concentration and initial concentration after $24 \mathrm{~h}$. Using following formula given by Gupta et al (2009)

$$
S_{\mathrm{e}}=\left(C_{0}-C_{\mathrm{e}}\right) * \frac{V}{M},
$$

where $S_{e}=$ equilibrium sorption concentration $(\mathrm{mg} / \mathrm{g}) ; C_{0}=$ initial solute concentration $(\mathrm{mg} / \mathrm{L})$; $C_{e}=$ equilibrium sorption concentration $(\mathrm{mg} / \mathrm{L}) ; V=$ volume of solution $(\mathrm{mL}) ; M=$ Mass of adsorbing material $(\mathrm{g})$.

After $24 \mathrm{~h}$ of sampling, $5 \mathrm{ml}$ aliquots were sampled from the glass tube filtered and then analysed for fluoride concentration. A standard curve was prepared in HACH DR5000 spectrophotometer, fluoride is tested at $570 \mathrm{~nm}$ wavelength. The estimated values of distribution coefficients for different isotherms are listed in table 2.

The above value of equilibrium sorption coefficient is used as independent input parameters. The transport of reactive solute depends upon the sorption characteristics of material and that is governed by equilibrium sorption coefficient. 
Table 2. Estimated values of equilibrium sorption coefficient for linear isotherm.

\begin{tabular}{lcc}
\hline Isotherm & Fine sand & Silt \\
$\left(K_{a}\right)$ & $\left(K_{n}\right)$ \\
\hline Linear isotherm & $0.00161 \mathrm{l} / \mathrm{gm}$ & $0.012561 / \mathrm{gm}$ \\
\hline
\end{tabular}

\section{Experimental procedure}

The laboratory model consists of a Plexiglas box, having dimensions of $4 \mathrm{~m}$ in length, $1 \mathrm{~m}$ wide and $1 \mathrm{~m}$ deep as shown in figure $2 \mathrm{a}$. Figure $2 \mathrm{~b}$ represents cross-section view of experimental

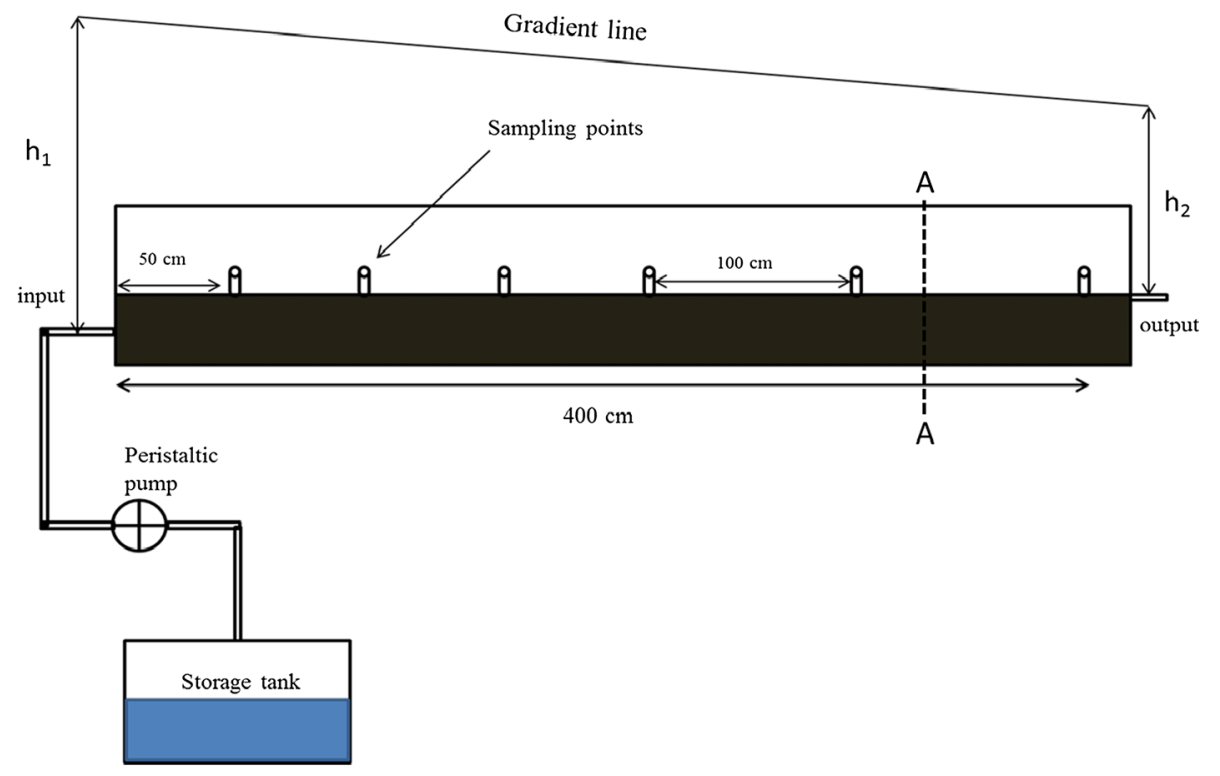

(a)

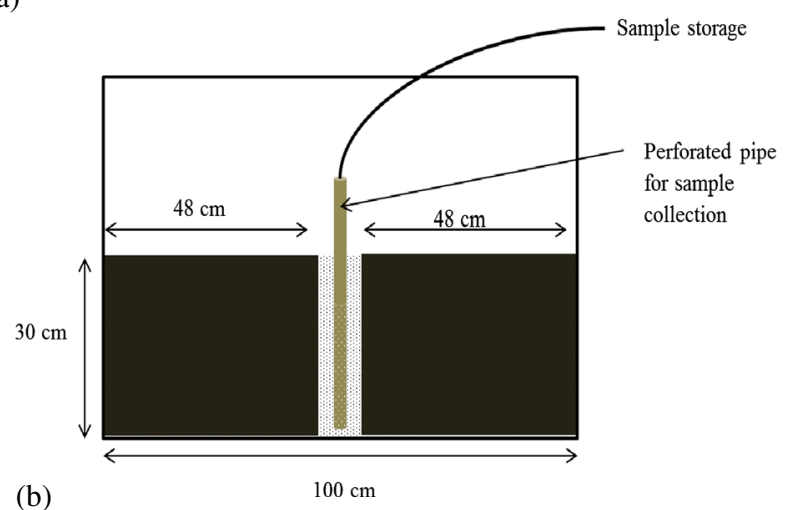

Figure 2. (a) Experimental set-up of stratified porous media model. (b) Cross section view of stratified porous media model. 
model as shown in figure 1a and thickness of the Plexiglas plate is $2 \mathrm{~mm}$. The influent and effluent end sides have been provided with hollow tubes at regular distance to facilitate the flow of water containing non-reactive and reactive tracers in experimental model. Tap water was tested before performing the experiments to measure the concentration of free chloride and fluoride. After measuring the concentration of tap water, a large size of storage tank was used to store the prepared solution of known concentration.

The experimental set-up as shown in figure $2 \mathrm{a}$ and $\mathrm{b}$, the width of silt layer is of $48 \mathrm{~cm}$ and a $4 \mathrm{~cm}$ of sand layer is sandwitched in between silt layers. The sand layer provides advective zone for facilitating the flow of fluid. Silt layer provides the immobile formation in which the mass transfer takes place due to diffusion only. The silt and sand layers together represent mobileimmobile regions, and induce physical non-equilibrium in the stratified porous media. Sampling points are located at regular interval to collect the samples at distributed location.

The bulk density of fine sand and silt were measured to be equal to $1.82 \mathrm{~g} / \mathrm{cm}^{3}$ and $1.63 \mathrm{~g} / \mathrm{cm}^{3}$, respectively. The porosity of the fine sand and silt were found to be 0.32 and 0.4 , respectively. After the complete saturation, a trial set of observation was done before actual experiment in which the tap water is introduced and eluted through fine sand layer and measurements were done. Initially, some concentration of chloride and fluoride appeared in the fraction collected. The concentration of chloride in tap water was observed as $35.5 \mathrm{mg} / \mathrm{L}$ through the silver nitrate titration method and $0.2 \mathrm{mg} / \mathrm{L}$ concentration of fluoride was measured with Hatch DR5000 spectrophotometer using SPADNS method.

Now in the actual sets of experiment, with water containing pre-defined concentration of tracer is screened through the fine sand layer only. A reservoir at the influent end was kept for the bulk storage of the displacing fluid, i.e., water containing for a known concentration of the tracer. Initially, conservative tracer such as chloride was used in the first experiment. A peristaltic pump at influent end was used to generate a constant head gradient to facilitate the flow and pump was set to operate on low flow velocity. An arrangement was made to distribute the flow in the fine sand layer through porous pipe, with a diameter of $3 \mathrm{~cm}$.

All the collected samples were stored in an air tight bottles and protected from direct sunlight. After the sampling, each sample was labelled and tested. For measuring chloride in the fraction collected, all the samples were titrated with potassium chromate as an indicator and silver nitrate as titrate. For the measurement of fluoride, spectrophotometer was used with the SPADNS method. Each of the $10 \mathrm{ml}$ sample mixed with $2 \mathrm{ml}$ of SPADNS dye and was provided 1 minute of reaction time, then small vials were filled with mixture and the absorbance of the sample was observed using the instrument. This absorbance was then converted into the concentration using standard concentration-absorbance profile plotted for the range of $10 \mathrm{ppm}$. Each of the standard solution of fluoride for the test prepared was within the range of concentration-absorbance profile plot.

\section{Effect of distance dependent coefficients on breakthrough curves}

In this section, the developed solution was used to represent the experimental data incorporating distance dependent dispersivity function. For estimating the parameters, firstly the simulation of experimental data was done for non-reactive tracer $\left(\mathrm{Cl}^{-}\right)$for which adsorption and degradation was zero. The estimation of conservative solute transport parameters was done and the value of first order mass transfer coefficient was obtained (table 3 ). The sensitivity of the distance dependent function parameters $\left(k, a_{1}, b_{1}\right)$ was studied to understand the distribution of breakthrough curves. Pore water velocity, $v_{a}=1.008 \mathrm{~m} /$ day, pulse duration, $T_{0}=3.4$ days, were considered 
Table 3. Parameters and estimated values of coefficient determination and root mean square error for breakthrough curve at $3 \mathrm{~m}$ down gradient distance.

\begin{tabular}{llcccc}
\hline & & $\begin{array}{c}\text { First order mass } \\
\text { transfer coefficient } \\
(\omega)\end{array}$ & Porosity & $\mathrm{R}^{2}$ & RMSE \\
\hline MIMC & Parameters & & & 0.97 & 0.062 \\
MIML & $\mathrm{k}=0.05$ & 0.2076 day $^{-1}$ & $\theta_{a}=0.33$, & 0.98 & 0.041 \\
MIME & $\mathrm{a}_{1}=1.0 \mathrm{~m}, \mathrm{~b}_{1}=0.1 / \mathrm{m}$ & & $\theta_{n}=0.4$ & 0.99 & 0.037 \\
\hline
\end{tabular}

to analyse the impact of various coefficient associated with different scale dependent functions at $3 \mathrm{~m}$ down gradient distance. The effects of the linear and exponential distance dependent coefficients were shown in figures 3-5.

In figure 3 where dispersivity is considered to be a linear function of $\mathrm{x}$ which is the distance from the source, it is clear that a higher $k$ value shows a highly dispersive transport. It can also be concluded that if the length of medium is considered infinity, dispersivity in that medium will become infinity which is not practical. A range of realistic values for $\mathrm{k}$ is suggested by Huang et al (1996) and assumed as $0 \leq \mathrm{k} \leq 1$. With a larger $\mathrm{k}$ value breakthrough time is reduced and a greater dispersive transport is observed.

For exponential distance dependent dispersivity, higher the value of $a_{1}$ and $b_{1}$ show the larger dispersion and the effect of $a_{1}$ and $b_{1}$ are similar in nature. In figure 4, the impact of coefficient $a_{1}$ is shown while fixing the value of coefficient $b_{1}$. It is seen that a lower peak and early breakthrough curves are the visible effect of the increasing value of $a_{1}$. However, in case of figure 5, the value of coefficient $\mathrm{a}_{1}$ is remained constant and value of $b_{1}$ varied from 0.01 to $0.5 \mathrm{~m}^{-1}$.

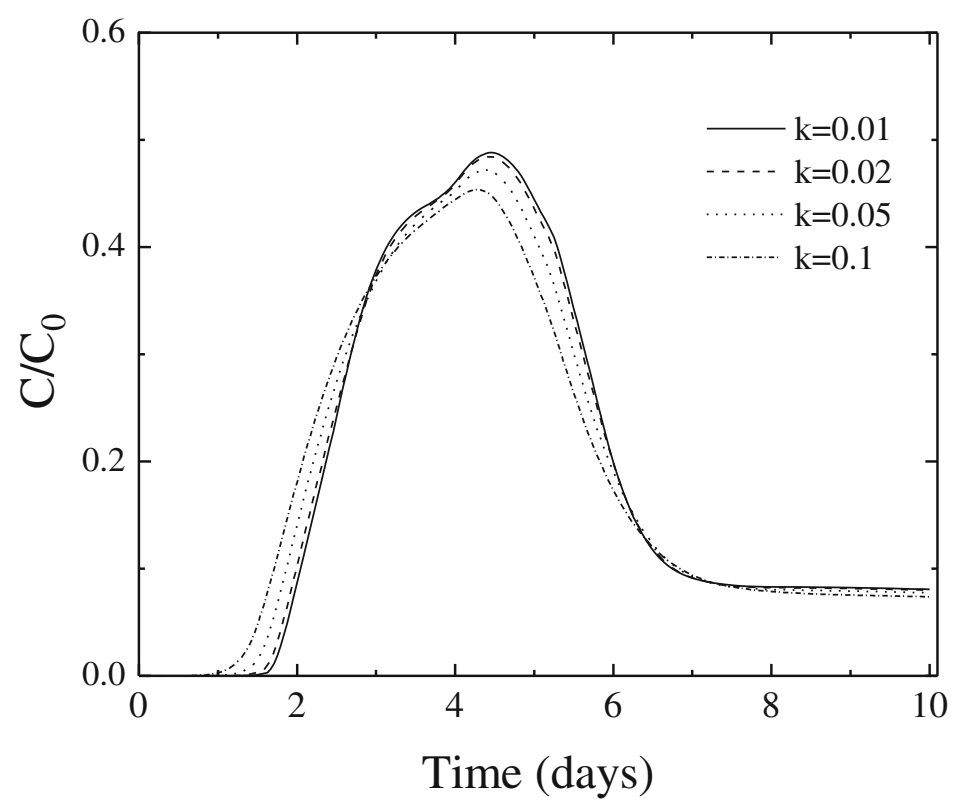

Figure 3. Breakthrough curves predicted at $3 \mathrm{~m}$ down gradient distance with different values of linear distance dependent coefficient. 


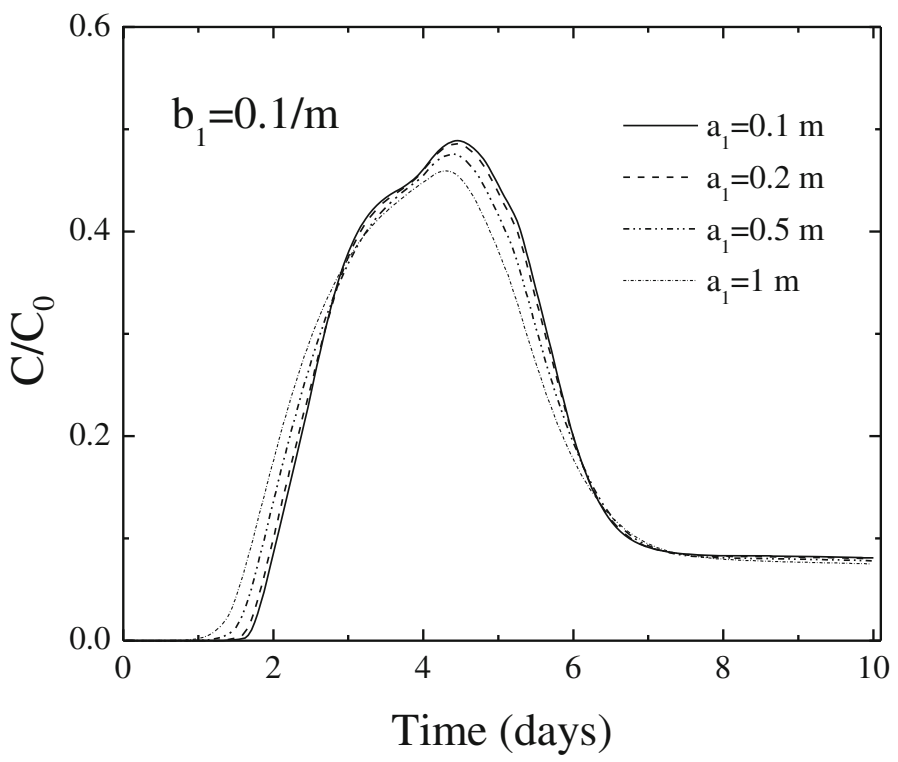

Figure 4. Breakthrough curves predicted at $3 \mathrm{~m}$ down gradient distance with different values of exponential coefficient, $a_{1}$.

It is clear from the comparison of figures 4 and 5 that the value of $b_{1}$ is more influencing the dispersivity in breakthrough curves. The advantage of exponential distance dependent dispersivity function is that even in infinite distance the value of dispersion will reach a numerically

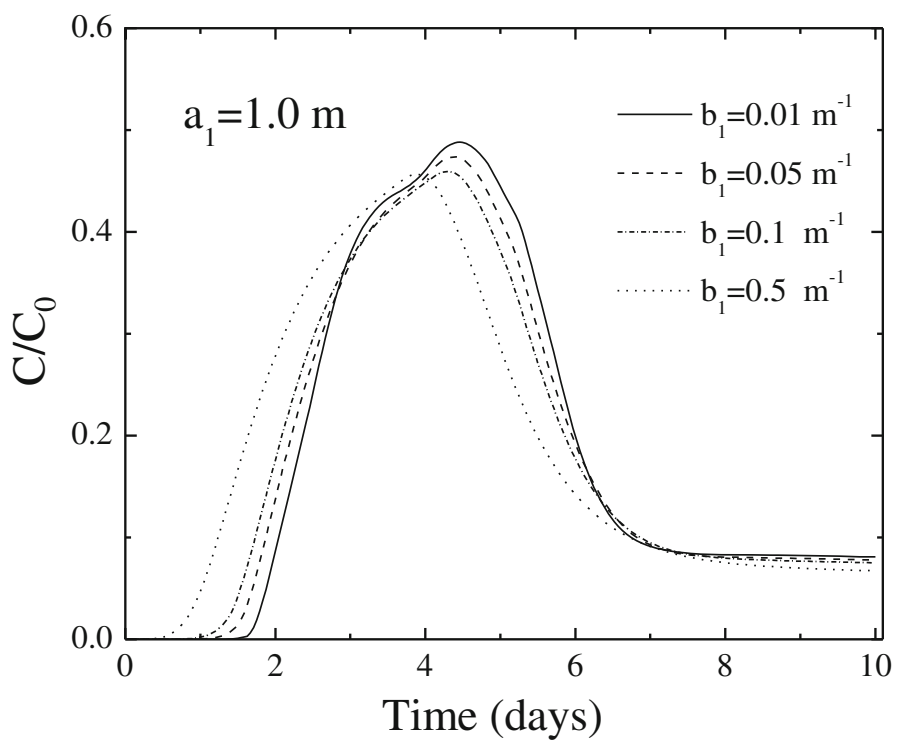

Figure 5. Breakthrough curvespredicted at distance of $3 \mathrm{~m}$ down gradient distance with different values of exponential coefficient, $b_{1}$. 
measurable value unlike linear distance dependent dispersivity function (Aral \& Liao 1996; Logan 1996; Hunt 1998).

\section{Simulation of experimental breakthrough curves of chloride using MIM model}

The estimation of the transport parameters are done by calibrating the non-reactive solute at $3 \mathrm{~m}$ down gradient distance available in the model. The experiments were performed by introducing low flow transport condition and flow velocity was measured as $1.008 \mathrm{~m} /$ day, pulse time was 3.4 day through sand and other physical parameters, i.e., porosity and hydraulic conductivity were already described in table 1 . The estimation of parameters was done in two parts; in first part the mass transfer coefficient $(w)$ was estimated by simulating the observed data of non-conservative solute $\left(\mathrm{Cl}^{-}\right)$. In second part, the estimation of the distance dependent dispersivity function's coefficient was done and values of coefficient of determination and root mean square error were shown in table 3 and by figure 6.

Figure 6 shows the breakthrough curve of chloride transport at $3 \mathrm{~m}$ down gradient distance, for which the mobile-immobile model (MIM) parameters and distance dependent dispersion coefficients were estimated.

The value of dispersivity equal to $0.3 \mathrm{~m}$ was used in the case of constant dispersion model. The estimated value of dispersivity was equal to $0.15 \mathrm{~m}$ was observed in case of linear distance dependent dispersion model. While in case of exponential dispersion model, the value of dispersivity was equal to $0.259 \mathrm{~m}$ corresponding to breakthrough curves observed at distance of $3 \mathrm{~m}$. The mobile-immobile exponential distance dependent dispersion model (MIME) was found to be a valid representation of the transport of chloride through the stratified medium. It is shown that the value of RMSE $=0.062$ and coefficient of determination $\mathrm{R}^{2}=0.97$ in case of mobileimmobile constant dispersion model (MIMC), and the value of RMSE $=0.041$ and $\mathrm{R}^{2}=0.98$ in case of mobile-immobile linear distance dependent dispersion model (MIML). In case of MIME

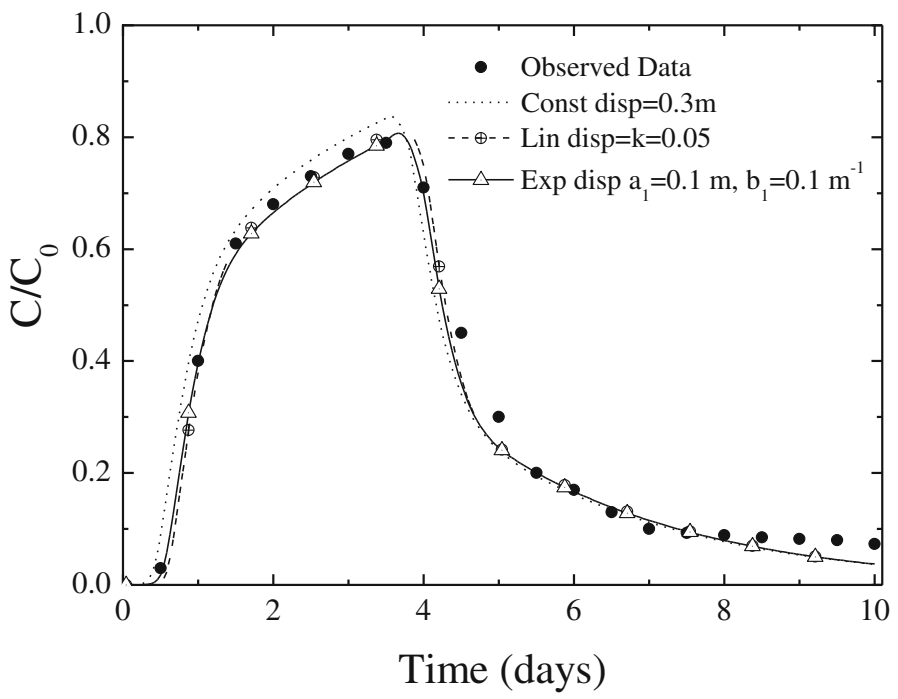

Figure 6. Breakthrough curves of chloride predicted at $3 \mathrm{~m}$ down gradient distance and simulated using various dispersion models. 
model, the value of RMSE $=0.037$ and coefficient of determination was $\mathrm{R}^{2}=0.99$. From above observation it can be stated that the value of coefficient of determination and root mean square error was smaller in case of MIME model when compared to both MIMC and MIML models. It was seen that MIML model also displayed a good accuracy in prediction but the infinitely increasing property of function with distance limits the use at large distances.

The simulation was primarily done for chloride transport at $3 \mathrm{~m}$ distance and the estimated values of transport parameters were shown in table 3 . The observed data of experimental breakthrough curves at preceding lengths (i.e., 0.5, 1, 1.5 and $2 \mathrm{~m}$ ) are simulated using the obtained parameters. Figures 7 to 10 show the simulation of experimental breakthrough curves predicted for the preceding lengths of $0.5,1,1.5$ and $2 \mathrm{~m}$.

Figures 7 and 8 show the simulation of experimental breakthrough curves predicted at down gradient distance of $0.5 \mathrm{~m}$ and $1 \mathrm{~m}$ using MIMC, MIML and MIME models. It is seen that the value of RMSE is smaller in case of MIML model as compared to MIMC and MIME models as shown in table 4. Hence, MIML model better fits the experimental breakthrough curve at shorter distances of $0.5 \mathrm{~m}$ and $1 \mathrm{~m}$, respectively. Estimated value of RMSE approximately remains same for MIML and MIME models for experimental breakthrough curves predicted at down gradient distance of $1.5 \mathrm{~m}$ (figure 9). Hence, linear distance and exponential distance dependent dispersion model show equal amount of accuracy for simulation of experimental breakthrough curves as shown in figure 9. However, mobile-immobile exponential dispersion model (MIME) gives best fit of the experimental breakthrough curves predicted at $2 \mathrm{~m}$. It is observed that the dispersivity after $1.5 \mathrm{~m}$ down-gradient distance turned in an exponential function of distance. Estimated value of correlation coefficient and root mean square error is shown in table 4.

It is clear that exponential dispersion function gives the better simulation of experimental breakthrough curves predicted at 2 and $3 \mathrm{~m}$, respectively. Linear dispersion function also

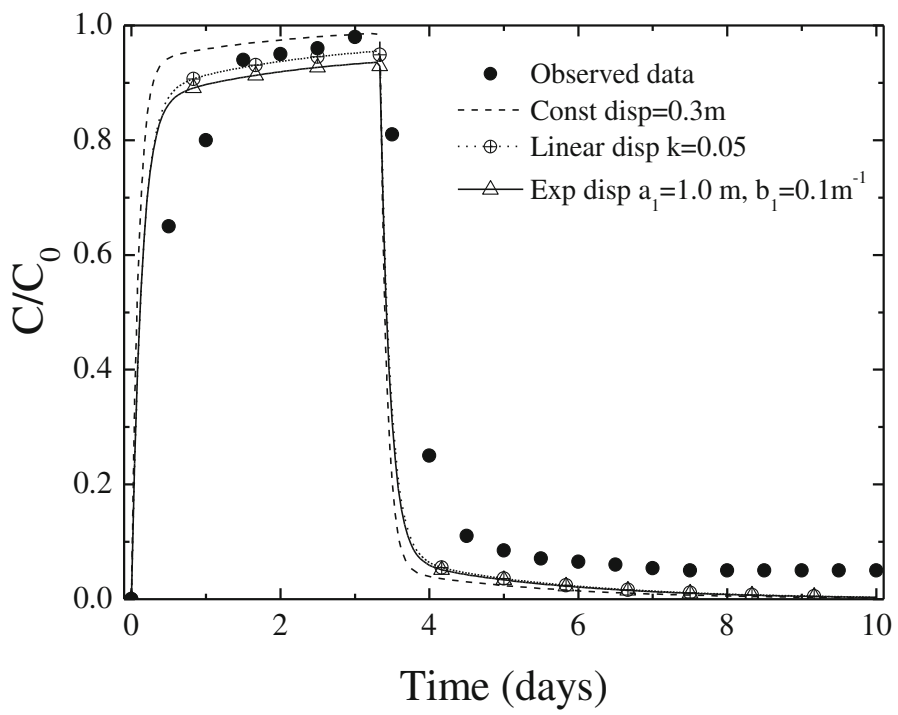

Figure 7. Breakthrough curves of chloride predicted at $0.5 \mathrm{~m}$ down gradient distance and simulated using constant dispersion, linear distance and exponential distance dependent dispersion models. 


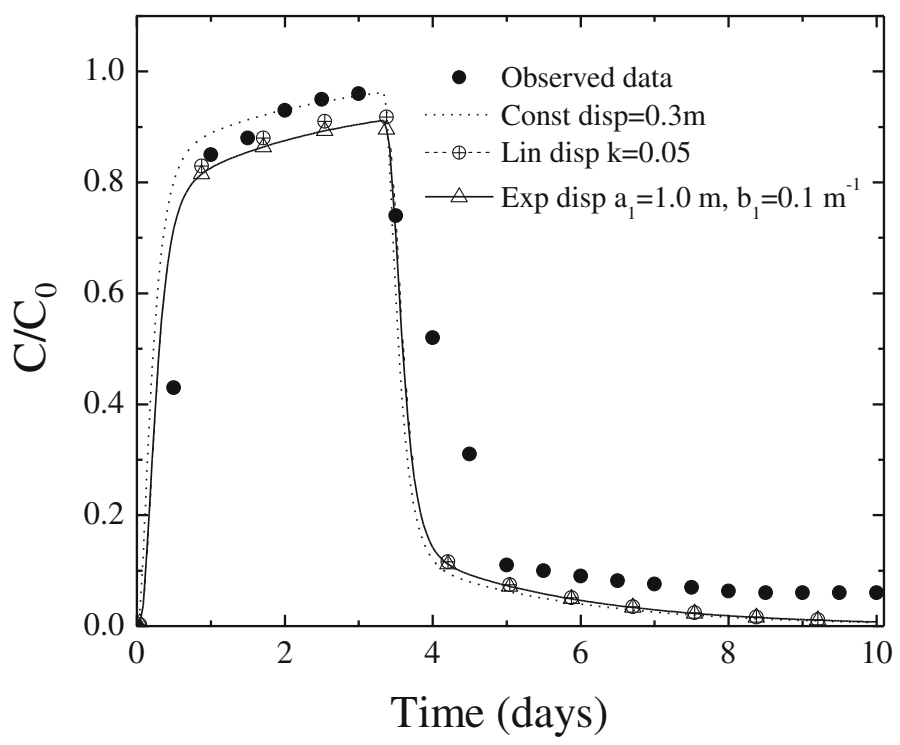

Figure 8. Breakthrough curves of chloride predicted at $1 \mathrm{~m}$ down gradient distance and simulated using constant dispersion, linear distance and exponential distance dependent dispersion models.

represents the nature of breakthrough curves closely, but the assumption of infinite dispersion at infinite distance is not applicable. If the linear dispersion function is calibrated for $0.5 \mathrm{~m}$ and prediction is made for $3 \mathrm{~m}$, it may overestimate the dispersivity.

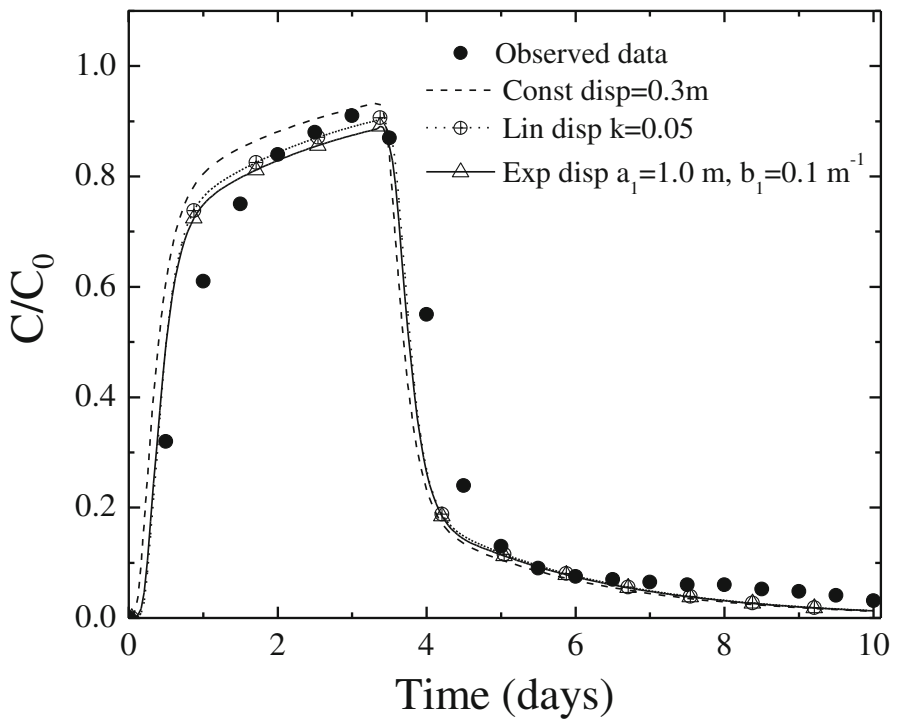

Figure 9. Breakthrough curves of chloride predicted at $1.5 \mathrm{~m}$ down gradient distance and simulated using constant dispersion, linear distance and exponential distance dependent dispersion models. 


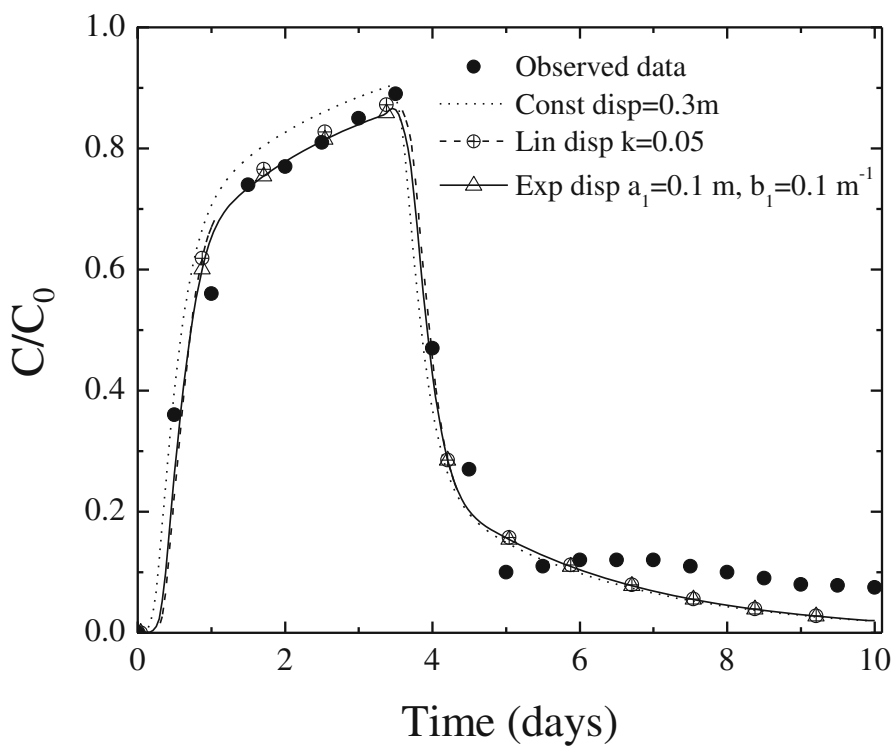

Figure 10. Breakthrough curves of chloride predicted at $2 \mathrm{~m}$ down gradient distance and simulated using constant dispersion, linear distance and exponential distance dependent dispersion models.

\section{Simulation of experimental breakthrough curves of fluoride}

In this section, multiprocess non-equilibrium transport process (MPNE) model is used to simulate the experimental breakthrough curves of fluoride. The estimation of MPNE parameters were done, while fixing the values of coefficient of distance dependent dispersion function obtained by non-reactive solute i.e., $\left(k, a_{1}, b_{1}\right)$. The input values of parameters i.e., pore water velocity $v_{a}=1.008 \mathrm{~m} /$ day, pulse time, $T_{0}=3.4$ days, $\theta_{a}=0.32, \theta_{n}=0.4$, mass transfer constant, $\omega=$ 0.2076 day $^{-1}$, bulk density, $\rho=1.898 \mathrm{~g} / \mathrm{cm}^{3}$, equilibrium sorption coefficient, $K_{a}=0.00161$ $1 / \mathrm{gm}, K_{n}=0.01261 \mathrm{l} / \mathrm{gm}$, first order reverse sorption coefficient, $k_{a 2}=k_{n 2}=0.12$ per day are used for simulation of experimental breakthrough curves. The values of MPNE parameters are shown in table 5. The linear isotherm considers that solid matrix has infinite sorption capacity and sorbed concentration increases with solute concentration. The value of equilibrium sorption coefficient is calculated by linear isotherm used as an independent input. Figure 11 shows

Table 4. Estimated value of correlation coefficient $\left(\mathrm{R}^{2}\right)$ and root mean square error (RMSE) for chloride transport in stratified media.

\begin{tabular}{|c|c|c|c|c|c|c|}
\hline \multirow[b]{2}{*}{ Distance (m) } & \multicolumn{2}{|c|}{ MIMC } & \multicolumn{2}{|c|}{ MIML } & \multicolumn{2}{|c|}{ MIME } \\
\hline & $\mathrm{R}^{2}$ & RMSE & $\mathrm{R}^{2}$ & $\overline{\text { RMSE }}$ & $\mathrm{R}^{2}$ & RMSE \\
\hline 0.5 & 0.813 & 0.167 & 0.881 & 0.133 & 0.875 & 0.137 \\
\hline 1 & 0.846 & 0.142 & 0.886 & 0.122 & 0.882 & 0.124 \\
\hline 1.5 & 0.889 & 0.112 & 0.934 & 0.086 & 0.934 & 0.087 \\
\hline 2 & 0.960 & 0.086 & 0.968 & 0.077 & 0.973 & 0.071 \\
\hline 3 & 0.973 & 0.062 & 0.983 & 0.039 & 0.989 & 0.040 \\
\hline
\end{tabular}


Table 5. Values of input parameters used for MPNE transport model.

\begin{tabular}{ll}
\hline Parameters & Values \\
\hline Pore velocity, $v_{a}$ & $1.008 \mathrm{~m} /$ day \\
$\theta_{a}$ & 0.32 \\
$\theta_{n}$ & 0.4 \\
$K_{a}$ & $0.00161 \mathrm{l} / \mathrm{gm}$ \\
$K_{n}$ & $0.01261 \mathrm{l} / \mathrm{gm}$ \\
$\mathrm{k}_{a 2}=\mathrm{k}_{n 2}$ & 0.12 day $^{-1}$ \\
$\mathrm{~F}_{a}=\mathrm{F}_{n}$ & 0.42 \\
$f$ & 0.45 \\
Dispersivity & $0.3 \mathrm{~m}$ \\
Distance dependent coefficient, $k$ & 0.05 \\
Exponential distance dependent coefficients $\mathrm{a}_{1}$ & $\mathrm{a}_{1}=1.0 \mathrm{~m}^{-1}$ \\
& $\mathrm{~b}_{1}=0.1 \mathrm{~m}^{-1}$ \\
\hline
\end{tabular}

the simulation for reactive solute assuming linear sorption isotherm and distance dependent dispersion at $3 \mathrm{~m}$ down gradient distance, for which MPNE parameters are obtained. It is shown that the value of correlation coefficient, $\left(\mathrm{R}^{2}=0.94\right)$ for exponential dispersion model is higher than constant and linear distance dependent dispersion models. The values of correlation coefficient, $\mathrm{R}^{2}=0.93$, root mean square error, $\mathrm{RMSE}=0.04$ in case of multiprocess non-equilibrium with constant dispersion model (MPNEC), $\mathrm{R}^{2}=0.89$, RMSE $=0.05$ in case of multiprocess non-equilibrium with linear distance dependent dispersion model (MPNEL), $\mathrm{R}^{2}=0.94$, RMSE $=0.04$ in case of multiprocess non-equilibrium with exponential dispersion model (MPNEE) are estimated. However, the value of root mean square error is smaller in case of exponential dispersion model as compared to linear dispersion model.

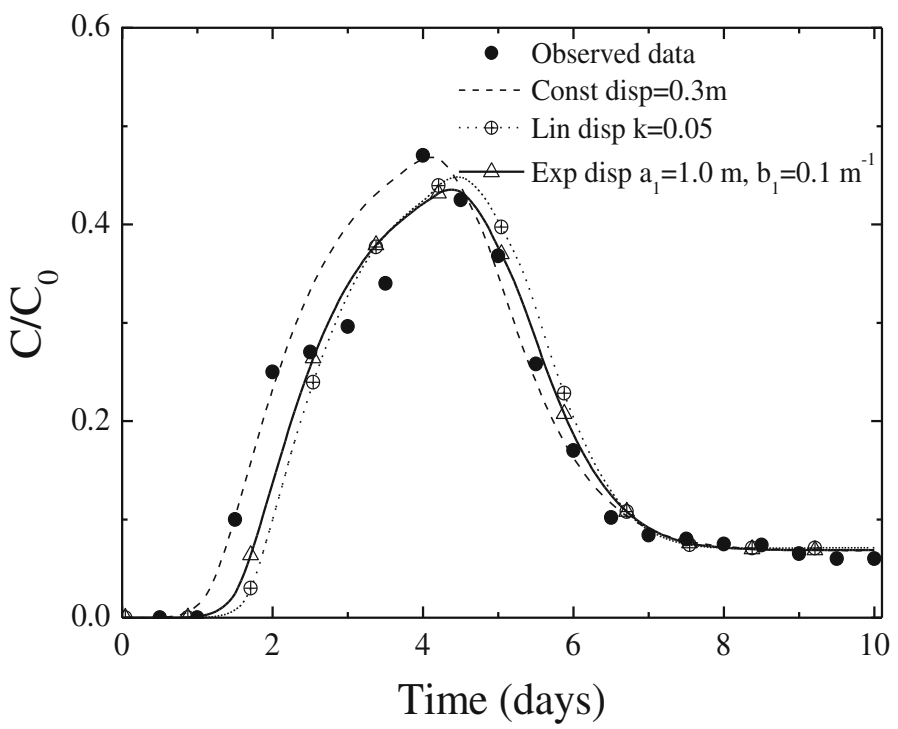

Figure 11. Breakthrough curves of fluoride predicted at $3 \mathrm{~m}$ down gradient distance and simulated using constant dispersion, linear distance and exponential distance dependent dispersion models. 


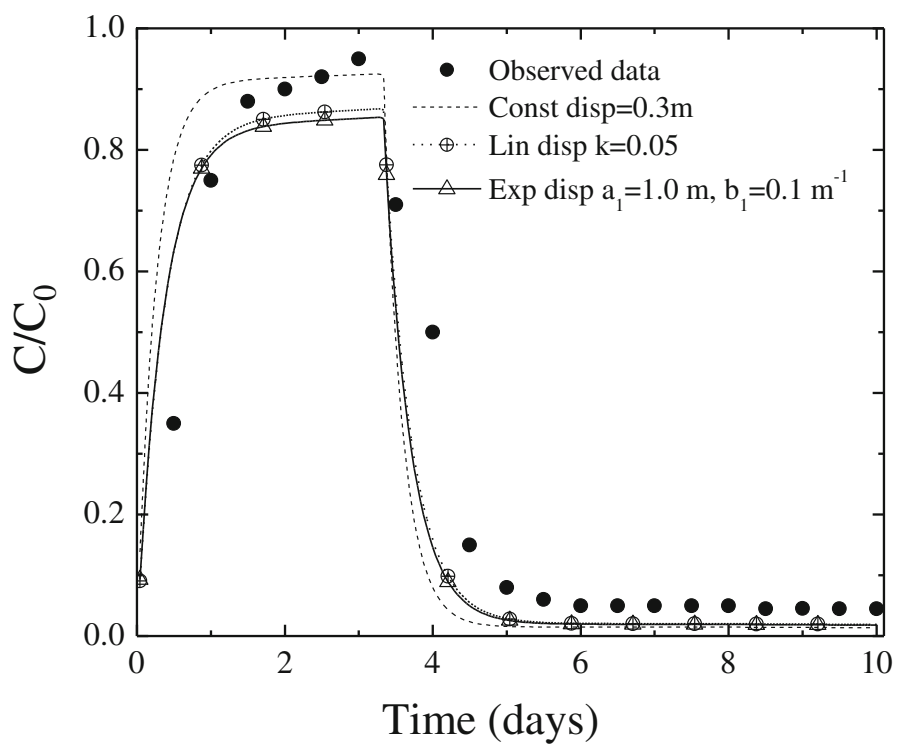

Figure 12. Breakthrough curves of fluoride predicted at $0.5 \mathrm{~m}$ down gradient distance and simulated using constant dispersion, linear distance and exponential distance dependent dispersion models.

Numerical simulation has been done for experimental breakthrough curves of fluoride at $3 \mathrm{~m}$ down gradient distance. It is seen that MPNEE and MPNEC model show the same accuracy in simulation of breakthrough curves. Estimated value of transport parameters are used for simulation of experimental breakthrough curves at smaller down gradient distance in stratified porous

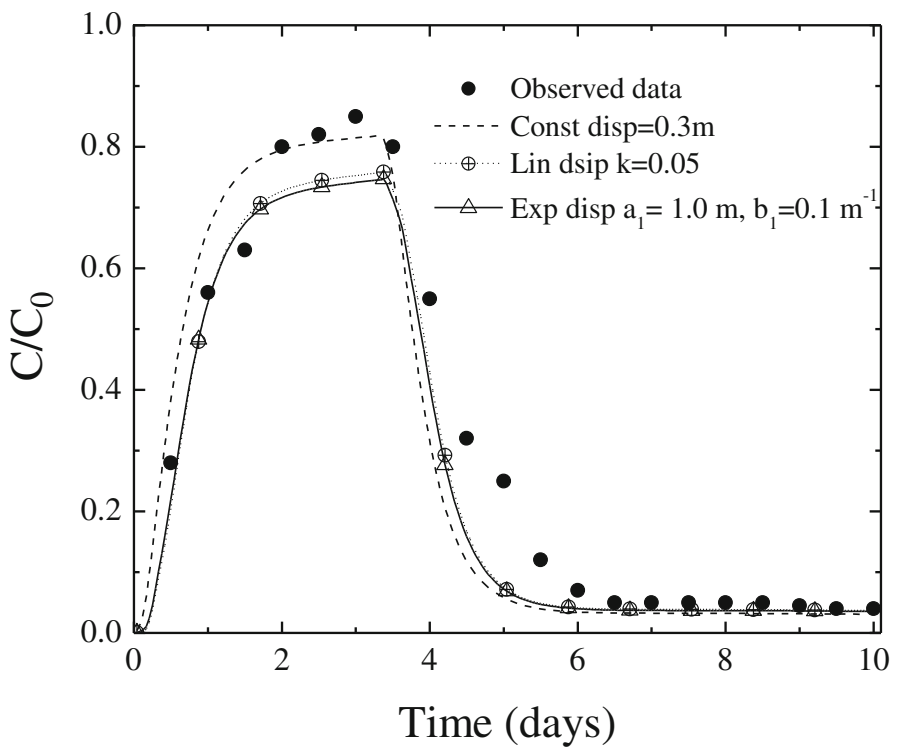

Figure 13. Breakthrough curves of fluoride predicted at $1 \mathrm{~m}$ down gradient distance and simulated using constant dispersion, linear distance and exponential distance dependent dispersion model. 


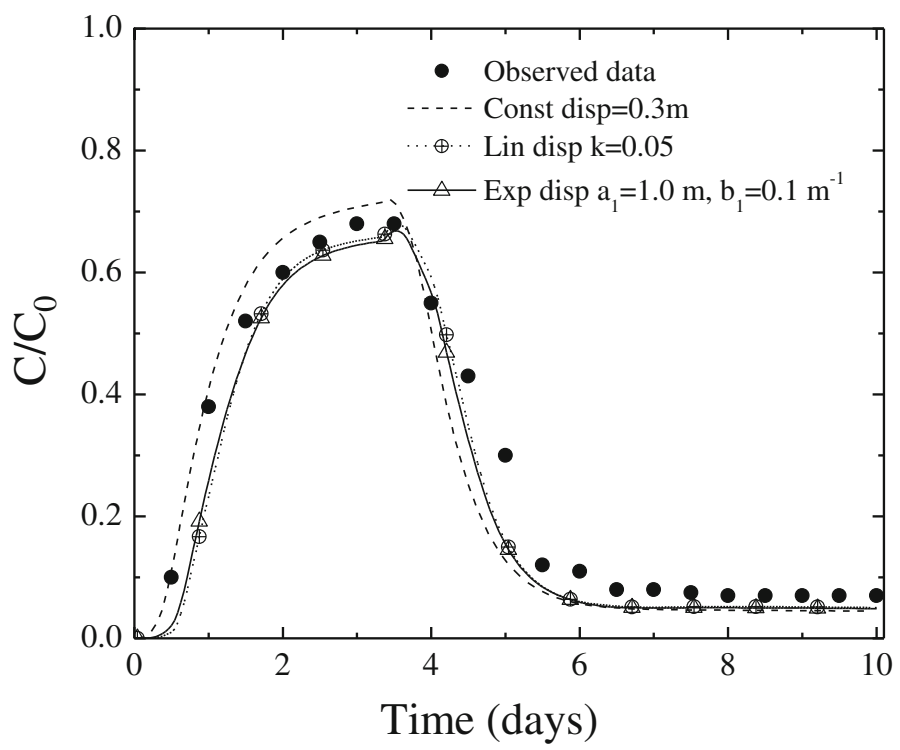

Figure 14. Breakthrough curves of fluoride predicted at $1.5 \mathrm{~m}$ down gradient distance and simulated using constant dispersion, linear distance and exponential distance dependent dispersion models.

model. Figures 12 to 15 show the observed and simulated breakthrough curves obtained by multiprocess non-equilibrium model with constant, linear and exponential distance dependent dispersion models.

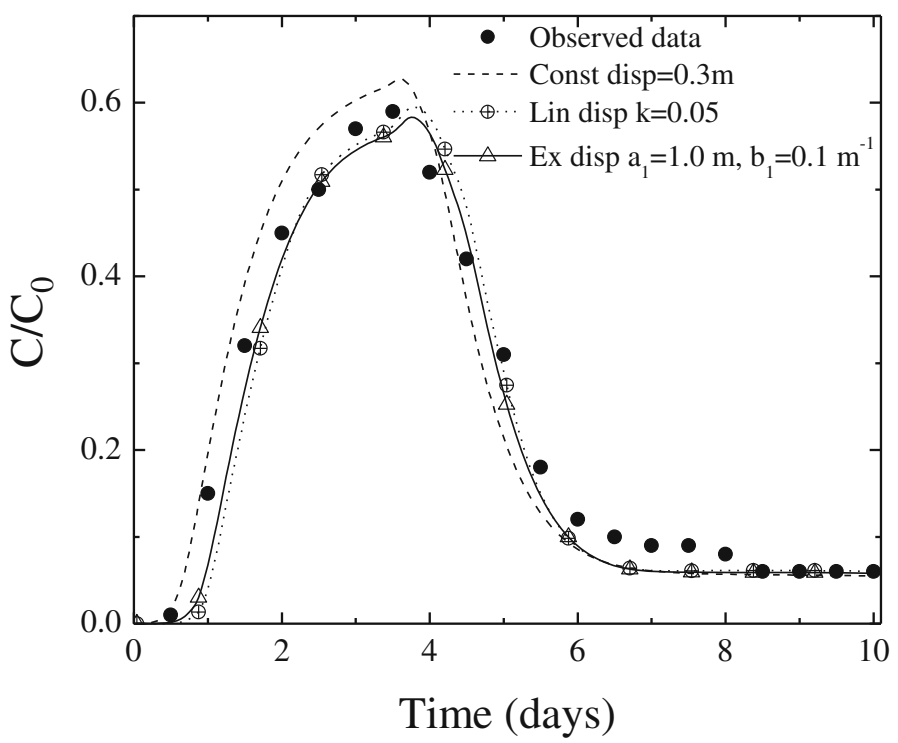

Figure 15. Breakthrough curves of fluoride predicted at $2 \mathrm{~m}$ down gradient distance and simulated using constant dispersion, linear distance and exponential distance dependent dispersion models. 
Table 6. Estimated value of correlation coefficient $\left(\mathrm{R}^{2}\right)$ and root mean square error (RMSE) for fluoride transport in stratified media.

\begin{tabular}{lccccccc}
\hline & \multicolumn{3}{c}{ RMSE } & & \multicolumn{3}{c}{$\mathrm{R}^{2}$} \\
\cline { 2 - 4 } Distance $(\mathrm{m})$ & MPNEC & MPNEL & MPNEE & & MPNEC & MPNEL & MPNEE \\
\hline 0.5 & 0.15 & 0.11 & 0.12 & & 0.82 & 0.9 & 0.89 \\
1 & 0.1 & 0.07 & 0.08 & & 0.9 & 0.94 & 0.93 \\
1.5 & 0.07 & 0.06 & 0.06 & & 0.93 & 0.94 & 0.94 \\
2 & 0.04 & 0.04 & 0.03 & & 0.95 & 0.96 & 0.97 \\
3 & 0.04 & 0.05 & 0.04 & & 0.93 & 0.89 & 0.93 \\
\hline
\end{tabular}

After analysing above breakthrough curves, it is observed that MPNEL displayed greater amount of accuracy in earlier distances i.e., at 0.5 and $1 \mathrm{~m}$ (figures 12 and 13). At distance of $1.5 \mathrm{~m}$ as shown in figure 14, the distribution of breakthrough curves simulated by MPNEL and MPNEE were same and at 1.5 and $2 \mathrm{~m}$ as shown in figures 10 and 15, the best simulation was given by MPNEE model. Table 6 shows the associated error obtained in observed and simulated curves, predicted by different constant dispersion and distance dependent dispersion models.

\section{Discussion}

Non-reactive transport was represented in figures 10-14, in which the numerical model was calibrated for the full length of system available and breakthrough curves at preceding length were predicted. It is clear that the transport of non-reactive solute is governed by mobile-immobile partitioning. Early appearance of concentration at $3 \mathrm{~m}$ shows strong dispersion and the nonzero nature of breakthrough curve shows the appearance of physical non-equilibrium. Calibration of model at $3 \mathrm{~m}$ length and the nature of breakthrough curve predicted at earlier lengths are wellaccepted. Table 4 shows the values of error associated and the simulation predicted by MIMC was adequately poor as compared to MIML and MIME models. The performance of MIML was better at earlier distances i.e., 0.5 and $1 \mathrm{~m}$. But at large distance, prediction was poor. The associated error in prediction using MIMC was adequately high at smaller distance in comparison to MIML and MIME. At smaller distances, MIML was found to give best fit of obtained BTC and at larger distances MIME was found to perform better simulation. At smaller distances MIML model was underestimating the dispersion coefficient, since the model was calibrated for large distance of $3 \mathrm{~m}$.

Figures 12-15 show the observed and simulated breakthrough curves for reactive solute transport under linear sorption assumption. The assumption of linear sorption isotherms were considered as a reflection of chemical non-equilibrium due to site-specific sorption. However, it was shown that the nature of breakthrough curve for reactive solute transport was more sensitive to site-specific sorption than the heterogeneity of medium (Abulaban \& Nieber 2000). The sorption was represented by linear isotherm and constant dispersion, linear and exponential distance dependent dispersion models were utilized in this study and the resulting error in prediction were shown in table 6.

In the simulation of reactive solute transport the same practices were followed. The model parameters were estimated for $3 \mathrm{~m}$ and prediction was made for previous distances. The model was calibrated in association of the values obtained by isotherm considered. In the experiment, the dissolved concentration of reactive solute $\left(\mathrm{Fl}^{-}\right)$was $5 \mathrm{PPM}$, which falls under the normal range of fluoride available in groundwater globally (Brindha \& Elango 2011). It is clear from 
the estimated values of root mean square error (RMSE) and correlation coefficient $\left(\mathrm{R}^{2}\right)$ that overall linear isotherm can provide fair representation of sorption dynamics in the micro aquifer system. For linear isotherm it is also found that the suitability of assumption of infinite sorption capacity for large distances for a limiting dissolved concentration is valid. By observing the mass distribution of breakthrough curve of reactive solute, it is clear that the system is under the influence of physical and chemical non-equilibrium. The tailing of BTC shows the mass sorbed on sorbent is desorbing and generally it takes infinite long time to attain a zero value. It is also clear that the assumption of local equilibrium is not applicable. Because of the transport, it is governed by a slower desorption process which is visible in form of the tailing of reactive solute.

\section{Conclusion}

The simulation for non-reactive and reactive solutes is done by MIM and MPNE model subsequently utilizing constant and distance dependent dispersivity functions. The experimental breakthrough curves were strongly dispersed for solute through stratified porous media. The MIM and MPNE model were calibrated for the largest distance of experimental set-up available and predicted breakthrough curves at previous distances. The nature of prediction for reactive and non-reactive solute obtained subsequently by MPNEL and MIML was better for 0.5 and $1 \mathrm{~m}$. At $1.5 \mathrm{~m}$ distance, the prediction by linear and exponential distance dependent function was found to be same for both reactive and non-reactive solute. It can be assumed as point of conflation, at which the simulation given by linear and exponential distance dependent dispersion model merges. An exponential distance dependent dispersion model simulates the experimental breakthrough curves predicted after $1.5 \mathrm{~m}$ down gradient onwards in the flow direction, accurately. It is clear that exponential distance dependent dispersivity is more suitable for large distances and linear or constant dispersivity function can be considered for small distances for simulating solute transport through stratified porous medium.

\section{References}

Abulaban A and Nieber J L 2000 Modeling the effects of nonlinear equilibrium sorption on the transport of solute plumes in saturated heterogeneous porous media. Advances in Water Resources 23(8): 893-905

Aral M M and Liao B 1996 Analytical solutions for two-dimensional transport equation with timedependent dispersion coefficients. J. Hydrol. Eng. 1(1): 20-32

Barry D A and Sposito G 1989 Analytical solution of a convection-dispersion model with time-dependent transport coefficients. Water Resour. Res. 25(12): 2407-2416

Brindha K and Elango L 2011 Fluoride in groundwater: Causes, implications and mitigation measures. Fluoride Properties, Applications and Environmental Management 111-136

Brusseau M L 1991 Application of a Multiprocess nonequilibrium sorption model to solute transport in a stratified porous medium. Water Resour. Res. 27(4): 589-595

Brusseau M L 1992 Transport of rate limited sorbing solute in heterogeneous porous media: Application of a one dimensional multifactor nonideality model to field data. Water Resour. Res. 28(9): 2485-2497

Brusseau M L and Rao P S C 1989 Sorption nonideality during organic contaminant transport in porous media. CRC Crit. Rev. Environ. Control 19(1): 33-99

Brusseau M L, Jessup R E and Rao P S C 1989 Modeling the transport of solutes influenced by multiprocessnon-equilibrium. Water Resour. Res. 25(9): 1971-1988

Burr D T, Sudicky E A and Naff R L 1994 Nonreactive and reactive solute transport in three-dimensional heterogeneous porous media: Mean displacement, plume spreading, and uncertainty. Water Resour. Res. 30(3): 791-815 
Chiou C T, Peters L J and Freed V H 1979 A physical concept of soil-water equilibria for non-ionic organic compounds. Science 206(4420): 831-832

Dykhuizen R C 1991 Asymptotic solutions for solute transport in dual velocity media. Mathematical Geology 23(3): 383-401

Elzein A H and Booker J R 1999 Groundwater pollution by organic compounds: a two-dimensional analysis of contaminant transport in stratified porous media with multiple sources of non-equilibrium partitioning. Int. J. Numerical and Analytical Methods in Geomech. 23(14): 1717-1732

Freeze R A and Cherry J A 1979 Groundwater Englewood Cliffs, N.J.: Prentice-Hall

Gao G, Zhan H, Feng S, Fu B, Ma Y and Huang G 2010 A new mobile-immobile model for reactive solute transport with scale-dependent dispersion. Water Resour. Res. 46(8): W08533, DOI: 10.1029/2009WR008707

Gerke H H and Van Genuchten M Th 1996 Macroscopic representation of structural geometry for simulating water and solute movement in dual-porosity media. Advances in Water Resour. 19(6): 343357

Gillham R W, Sudicky E A, Cherry J A and Frind E O 1984 An advection- diffusion concept for solute transport in heterogeneous-unconsolidated geological deposits. Water Resour. Res. 20(3): 369-378

Goltz N M and Roberts P V 1986 Three-dimensional solution for solute transport in an infinite medium with mobile and immobile zones. Water Resour. Res. 22(7): 1139-1148

Gupta M K, Singh A K and Srivastava R K 2009 Kinetic sorption studies of heavy metal contamination on Indian expansive soil. E-J. Chem. 6(4): 1125-1132

Huang H, Huang Q and Zhan H 2006 Evidence of one dimensional scale-dependent fractional advectiondispersion. J. Contam. Hydro. 85(1-2): 53-71

Huang K, Van Genuchten M T and Zhang R 1996 Exact solutions for one-dimensional transport with asymptotic scale-dependent dispersion. Appl. Math. Model. 20: 298-308

Hunt B 1998 Contaminant source solutions with scale-dependent dispersivities. J. Hydrologic Eng. 3(4): 268-275

Karickhoff S W 1981 Semi empirical estimation of sorption of hydrophobic pollutants on natural sediments and soils. Chemosphere 10(8): 833-846

Khan A A, Muthukrishnan M and Guha B K 2010 Sorption and transport modeling of hexavalent chromium on soil media. J. Hazardous Materials 174(1): 444-454

Logan L D 1996 Solute transport in porous media with scale-dependent dispersion and periodic boundary conditions. J. Hydrol. 184(3): 261-276

Liu Gang Si and Bing C 2008 Analytical modeling of one dimensional diffusion in layered systems with position dependent diffusion coefficients. Advances in water Resour. Res. 31(2): 251-268

Molz F J, Guven O and Melville J G 1983 An examination of scale-dependent dispersion coefficient. Ground Water 21(6): 715-725

Naik M S, Reddy H P P and Sivapullaiah P V 2008 A reliable method of obtaining breakthrough curves of ions in soils using transport equation. In The 12th International Conference of International Association for Computer Methods and Advances in Geomechanics (IACMAG) (pp. 1-6)

Pickens J F and Grisak G E 1981 Scale-dependent dispersion in a stratified granular aquifer. Water Resour. Res. 17(4): 1191-1211

Rao P S C, Jessup R E, Rolston D E, Davidson J M and Kilcrease D P 1980 Experimental and mathematical description of nonadsorbed solute transfer by diffusion in spherical aggregates. Soil Sci. Soc. Am. J. 44(4): 684-688

Roberts P V, Goltz M N and Mackay D M 1986 A natural gradient experiment on solute transport in a sand aquifer: 3. Retardation estimates and mass balances for organic solutes. Water Resour. Res. 22(13): 2047-2058

Sudicky E A, Gillham R W and Frind E O 1985 Experimental investigation of solute transport in stratified porous media, 1. The nonreactive case. Water Resour. Res. 21(7): 1035-1041

Starr R C, Gillham R W and Sudicky E A 1985 Experimental investigation of solute transport in stratified porous media, 2. The reactive case. Water Resour. Res. 21(7): 1043-1050 
Tang D H, Frind E O and Sudicky E A 1981 Contaminant transport in fractured porous media: Analytical solution for a single fracture. Water Resour. Res. 17(3): 555-564

Van Duijn C J and van derZee S E A T M 1986 Solute transport parallel to an interface separating two different porous materials. Water Resour. Res. 22(13): 1779-1789, DOI: 10.1029/WR022i013p01779

Van Genuchten M Th and Wierenga P J 1976 Mass transfer studies in sorbing porous media: Analytical solutions. Soil Sci. Soc. Am. J. 40(3): 473-479

Van Genuchten M Th 1981 Non-equilibrium transport parameters from miscible displacement experiments. Res. Rep. 119, U.S. Salinity Lab., U.S. Dep. of Agric. Washington, D.C

Xu L and Brusseau M L 1996 Semianalytical solution for solute transport in porous media with multiple spatially variable reaction processes. Water Resour. Res. 32(7): 1985-1991

Yates S R 1990 An analytical solution for one-dimensional transport in heterogeneous porous media. Water Resour. Res. 26(10): 2331-2338

Yates S R 1992 An analytical solution for one-dimensional transport in porous media with an exponential dispersion function. Water Resour. Res. 28(8): 2149-2154 